\title{
Papers
}

Editorial by Dutton and Cleary

\section{Randomised controlled trial of treatment of unilateral visual impairment detected at preschool vision screening}

M P Clarke, C M Wright, S Hrisos, J D Anderson, J Henderson, S R Richardson

\begin{abstract}
Objectives To test the efficacy of treatment for unilateral visual loss detected by preschool vision screening and the extent to which effectiveness varies with initial severity.

Design Randomised controlled trial of full treatment with glasses and patching, if required, compared with glasses only or no treatment. Masked assessment of best corrected acuity after one year of follow up.

Setting Eight UK eye departments.

Participants 177 children aged 3-5 years with mild to moderate unilateral impairment of acuity (6/9 to $6 / 36)$ detected by screening.

Results Children in the full and glasses treatment groups had incrementally better visual acuity at follow up than children who received no treatment, but the mean treatment effect between full and no treatment was equivalent to only one line on a Snellen chart $(0.11 \log$ units; $95 \%$ confidence interval 0.050 to $0.171 ; \mathrm{P}<0.0001)$. The effects of treatment depended on initial acuity: full treatment showed a substantial effect in the moderate acuity group (6/36 to $6 / 18$ at recruitment) and no significant effect in the mild acuity group $(6 / 9$ to $6 / 12$ at recruitment) ( $\mathrm{P}=0.006$ for linear regression interaction term). For 64 children with moderate acuity loss the treatment effect was $0.20 \mathrm{log}$ units, equivalent to one to two lines on a Snellen chart. When all children had received treatment, six months after the end of the trial, there was no significant difference in acuity between the groups.

Conclusions Treatment is worth while in children with the poorest acuity, but in children with mild (6/9 to 6/12) unilateral acuity loss there was little benefit. Delay in treatment until the age of 5 did not seem to influence effectiveness.
\end{abstract}

\section{Introduction}

Amblyopia is a form of cerebral visual impairment caused by abnormal vision, commonly uncorrected refractive error, during a sensitive period of development. ${ }^{1-4}$ Treatment is thought to be effective only during this sensitive period, which varies for different types of amblyopia but most commonly lasts until 7 years of age. ${ }^{5-7}$ The most common forms of amblyopia are monocular, due to squint (strabismic amblyopia), or refractive error, with a prevalence of $2-4 \%{ }^{8}{ }^{9}$ Such impairment is a bar to certain occupations, affects binocular vision and stereopsis, and causes considerable disability if the normal eye suffers trauma or disease. ${ }^{10}$

Strabismic amblyopia usually presents with a visible squint, but refractive amblyopia or a small angle strabismus may not be detected until it is too late for treatment to be effective. Preschool vision screening became widespread in the United Kingdom and
Europe during the 1970s and 1980s, with the aim of detecting unilateral amblyopia at a stage when treatment would be effective. ${ }^{11-16}$ Methods of screening and recommended referral criteria vary, ${ }^{17}$ though children are usually referred if acuity is $6 / 9$ or worse. ${ }^{18}$ Glasses alone may prevent or treat refractive amblyopia, ${ }^{19}$ but most children require additional treatment with patching $^{3}$ or penalisation of the normal eye (by blurring vision, usually with atropine drops).$^{20}$ This treatment was developed empirically, supported by animal studies, ${ }^{121}$ but has never been subjected to a randomised controlled trial.

Recent studies have raised concerns about the appropriateness of amblyopia as a target condition for early screening ${ }^{22}$ and the possible adverse psychological impact of treatment weighed against the limited disability it causes. ${ }^{23}$ A systematic review concluded that there was no robust evidence for the effectiveness of amblyopia treatment. ${ }^{15}$ Our study arose out of the controversy generated by that review. We looked at the effectiveness of treatment by patching plus glasses or glasses alone compared with no treatment and explored the extent to which effectiveness varied with initial severity.

\section{Methods}

The study was a pragmatic, single masked, randomised controlled trial in eight UK children's eye clinics. It was designed to assess the benefits of current standard treatment of children who fail preschool vision screening tests.

\section{Recruitment of participants}

In all centres preschool vision testing was already conducted by community based orthoptists. Children were referred to dedicated recruitment clinics if, after two standard screening tests, they had $6 / 6$ vision in one eye and $6 / 9$ to $6 / 36$ vision in the other. If the acuity findings were confirmed in the recruitment clinic, the child was eligible to join the trial. Consent was then requested from the carer by the trial centre ophthalmologist. If the ophthalmologist found any other ocular abnormalities the child was excluded from the trial. A training video of the recruitment procedure and trial protocol handbooks were supplied to all participating centres. At each research review an assessment form was completed and forwarded to the trial office.

\section{Allocation to treatment}

Once consent was obtained, the child was randomly allocated to a treatment group by the researchers phoning the trial centre, where allocation tickets, computer generated for each centre before the start of recruitment, were stored in numbered, sealed, opaque envelopes. The computer program produced random permuted block allocation with random swapping between blocks of length 4 
and 6 . Randomisation was stratified by trial centre and acuity for entry acuity of $6 / 9,6 / 12$ and $6 / 18$, but children with acuity $6 / 24$ and $6 / 36$ were randomised together across all centres.

\section{Treatment}

After randomisation, all children were tested for refractive error with cycloplegic drops to eliminate artefact due to accommodation. Glasses were dispensed to children only in the full and glasses groups, who were then seen after six weeks to verify the glasses prescription. Children in the full treatment group then started to wear a patch if their corrected acuity remained reduced. They were reviewed every six weeks and managed by the treatment orthoptist according to protocol. Children in the glasses group received no further treatment for 52 weeks. Children in the control group (no treatment) received no active treatment for 52 weeks. For those children receiving treatment, we assessed compliance using weekly parental diaries for the first 12 weeks of glasses wear and with daily diaries for the duration of patching treatment.

\section{Measurements}

A research orthoptist, who remained masked throughout to the child's treatment group, assessed vision at 24, 52, 54, and 78 weeks. If masking was breached, subsequent testing was performed by a deputy research orthoptist. At six months, if a child in the no treatment and glasses treatment groups developed manifest squint or acuity below 6/36 they were offered full treatment. As children in the no treatment group did not receive glasses until after the 52 week follow up, visual acuity testing was performed without glasses for all groups at 24 and 52 weeks. At the 52 week follow up children in all groups were prescribed glasses. Children in the no treatment group were instructed to begin wearing glasses only the day before the 54 week follow up, when best corrected (that is, without glasses) visual acuity was recorded.

Children in the no treatment and glasses treatment groups were then offered patching treatment as required with the same protocol. Six months later, 18 months after recruitment, we saw all the children once more and tested their best corrected visual acuity.

Analysis

Prestated outcome measures were uncorrected and corrected $\operatorname{logMAR}$ acuity, compared between the three groups at 52 and 54 week follow up. A planned subgroup analysis compared children whose acuity loss at recruitment was mild (6/12 to $6 / 9)$ with those whose acuity loss was moderate (6/36 to 6/18). LogMAR acuity was obtained with Glasgow acuity cards ${ }^{24}$ (marketed as LogMAR Crowded Test by Keeler, Windsor) and were used in preference to Snellen acuity values as logMAR is a continuous measure of acuity, more suited to statistical analysis. Change in acuity from recruitment was a secondary outcome measure for those able to perform logMAR testing at recruitment. Analysis was by intention to treat, with all children for whom follow up data were available analysed in their allocated groups. The main planned analytical method was trend in analysis of variance between treatment groups with linear regression used to fit the interaction term for the initial acuity group.

All acuities shown in this paper are crowded logMAR, and, unless otherwise stated, all follow up acuity data are best corrected acuity. Prior power calculations were limited by a lack of data but indicated that 50 children in each group would supply ample power to detect a clinically useful difference of one or more lines on a Snellen chart.

\section{Results}

Recruitment to the trial opened in April 1999 and closed in December 2000; 12 month follow up closed in December 2001 and 18 month follow up in June 2002. Of 490 children in eight trial centres thought to fulfil trial entry criteria at screening, 434 were seen at recruitment clinics, but 180 of these no longer met the trial entry criteria (of these, 113 had normal vision, 53 had reduced acuity in both eyes (fig 1). The parents of 77 children refused to participate, with higher rates of refusal among parents

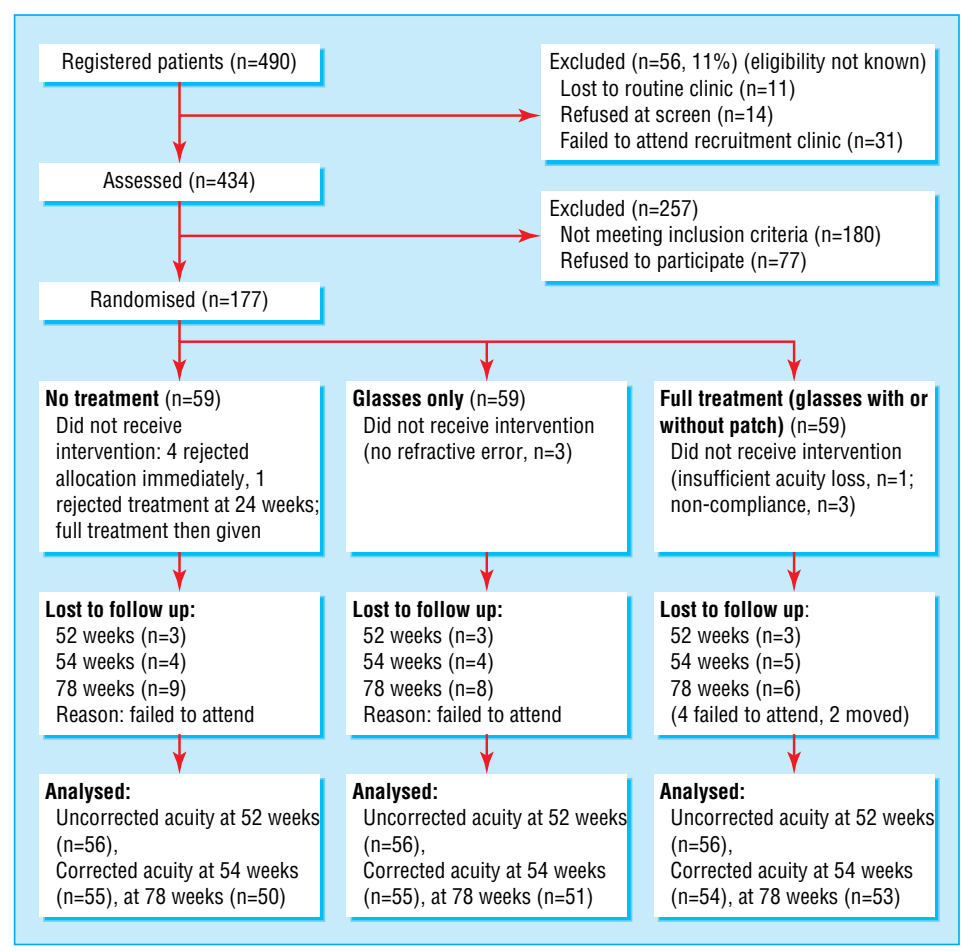

Fig 1 Participant flow through trial of treatment of visual impairment detected at preschool vision screening 


Table 1 Baseline data for all children who were eligible for the trial
\begin{tabular}{lccc} 
& Eligible & Refused & Recruited \\
\hline No of children & 254 & 77 & 177 \\
\hline $\begin{array}{l}\text { Mean (SD) age at recruitment } \\
\text { (months) }\end{array}$ & $47.7(4.9)$ & $47.4(4.3)$ & $48.1(5.0)$ \\
\hline No (\%) with Snellen acuity: & & & \\
\hline $6 / 9$ & $78(31)$ & $20(26)$ & $58(33)$ \\
\hline $6 / 12$ & $73(29)$ & $21(27)$ & $52(29)$ \\
\hline $6 / 18$ & $54(21)$ & $12(16)$ & $42(24)$ \\
\hline $6 / 24$ and 6/36 & $49(19)$ & $24(31)$ & $25(14)$ \\
\hline No (\%) with microtropia & $10(4)$ & $4(5)$ & $6(3)$ \\
\hline
\end{tabular}

Table 2 Baseline data at recruitment, by allocated treatment group. Values are number $(\%)$ unless otherwise stated

\begin{tabular}{lcccc} 
& No treatment & Glasses & Full & Total \\
\hline No of children & 59 & 59 & 59 & 177 \\
\hline Mean (SD) age (months) & $48.4(5.1)$ & $47.1(5.2)$ & $47.6(4.5)$ & $47.7(4.9)$ \\
\hline Snellen acuity: & & & & \\
\hline $6 / 9$ & 20 & 20 & 18 & $58(33)$ \\
\hline $6 / 12$ & 15 & 18 & 19 & $52(29)$ \\
\hline $6 / 18$ & 14 & 14 & 14 & $42(24)$ \\
\hline $6 / 24^{\star}$ & 7 & 3 & 2 & $12(7)$ \\
\hline $6 / 36^{*}$ & 3 & 4 & 6 & $13(7)$ \\
\hline Microtropia & 4 & 2 & 0 & $6(3)$ \\
\hline Anisometropia & 45 & 41 & 41 & $127(72)$ \\
\hline
\end{tabular}

${ }^{*}$ Randomised as one group.

of children with lower acuity. There were thus 177 children who joined the study at a mean age of 48.1 (SD 5.0) months (table 1) having been screened at a mean age of 45.6 (4.6) months. Sixty seven $(38 \%)$ children were in the moderate category. The median number of recruits per centre was 21.5 (range 5-52). The randomisation allocated 59 children to each group, with a reasonable distribution of acuity (table 2). Of the total, $173(98 \%)$ had a significant refractive error, $127(72 \%)$ of whom had anisometropia (a significant difference in refractive error between the two eyes) (table 2 ).

In the first six weeks, 74 (69\%) of the 107 children prescribed glasses wore them most or all of the time, with only six $(6 \%)$ not wearing them at all. In the full treatment group, vision in 13 with mild acuity loss and four with moderate loss improved to normal with glasses correction alone. The 42 remaining (71\%) required patching for a mean of 20 (SD 10) weeks. Twenty five wore their patch for two thirds or more of prescribed time, but three would not wear it at all (fig 1).

Five parents in the no treatment group rejected their allocation, four immediately and one after six months. The children then received active treatment; two of these subsequently defaulted from all follow up. No child required active treatment because of deterioration.

Follow up data at 52 weeks were available for $168(95 \%)$ children and at 54 weeks for 164 (93\%) (fig 1). Children in the full and glasses treatment groups had incrementally better uncorrected and corrected visual acuity at follow up compared with those in the no treatment group, but the overall treatment effect was small (table 3). However, at follow up only two (4\%) children in the full treatment group still had moderate acuity loss (worse than Snellen equivalent 6/12 (logMAR $<0.3)$ ) compared with $15(27 \%)$ in the untreated group $\left(\chi^{2}\right.$ trend $\left.\mathrm{P}=0.001\right)$.

Glasses produced a fairly fixed gain of around $0.08 \log$ units at all acuities, but the treatment effects differed depending on initial acuity (fig 2, table 4). Full treatment showed a substantial effect in the moderate acuity group and no significant effect in the mild acuity group $(\mathrm{P}=0.006$ for linear regression interaction term).
Change in acuity since recruitment showed similar treatment effects to those seen between treatment groups at 12 month follow up. The change in acuity for the no treatment group was a mean gain without glasses of 0.1 (SD 0.2) log units for the mild and 0.05 (SD 0.16 ) for the moderate initial acuity group. Preplanned analysis by centre showed no differences in the treatment effect after adjustment for initial acuity, which did differ between centres.

After 54 week follow up, children in the no treatment and glasses treatment groups received treatment according to the protocol for the full treatment group (table 5). In the no treatment group, seven (13\%) had normal acuity and received no treatment. Twenty four (44\%) achieved normal acuity with glasses correction only, and 24 (44\%) required patching in addition to glasses. In the glasses group, $20(36 \%)$ needed treatment with patching. In addition, seven children in the full group required retreatment with patching at this point.

At 78 week follow up, six months after the formal end point of the trial, $154(87 \%)$ children attended and there was no significant difference in acuity between the three treatment groups (tables 3 and 4).

\section{Discussion}

Previous studies in humans and animals have claimed that amblyopia can, with good compliance, be improved with treatment. ${ }^{20} 212526$ However, it is not known what level of loss of acuity merits treatment as no previous study has included an untreated control group and many have excluded children with milder acuity loss. We also specifically evaluated the treatability of children identified by standard screening programmes, which will inevitably also identify some children who are not truly amblyopic but who can be identified as such only in retrospect, after their acuity has returned to normal with glasses.

\section{Avoiding bias}

Our trial was designed to minimise possible sources of bias. Allocation to treatment was managed centrally, outcomes were assessed masked, and different testing methods were used for the trial to reduce practice effects. It was not feasible to test all children with the same frequency as those in the full treatment group, so we cannot rule out the possibility that these children may have become generally more proficient at vision testing. The analysis was by intention to treat, which could underestimate the true treatment effect. However, only five parents rejected allocation to no treatment and compliance was reasonable. The trial was amply

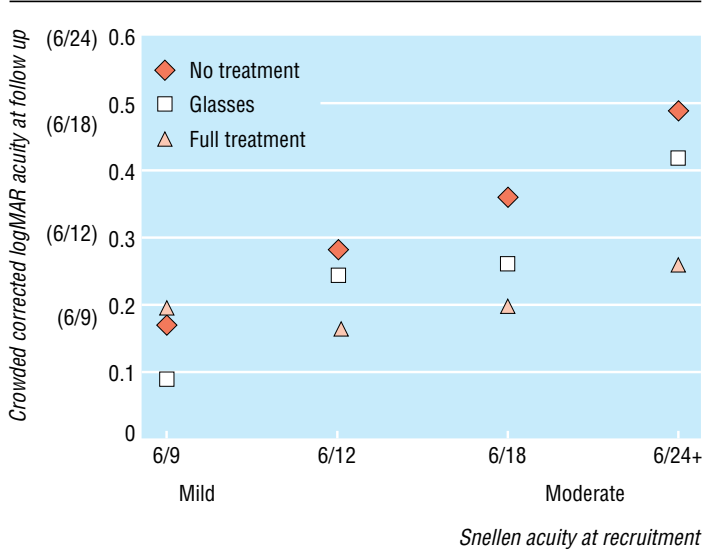

Fig 2 Corrected crowded logMAR acuity at 54 weeks by initial Snellen acuity level 
Table 3 Visual acuity after follow up to trial end point and six months after trial, by treatment group

\begin{tabular}{|c|c|c|c|c|c|c|}
\hline \multirow[b]{2}{*}{ LogMAR acuity } & \multirow{2}{*}{$\begin{array}{c}\text { No treatment } \\
\text { Mean }(\mathrm{SD}) \\
\text { logMAR }\end{array}$} & \multicolumn{2}{|c|}{ Glasses } & \multicolumn{2}{|c|}{ Full } & \multirow[b]{2}{*}{$\begin{array}{l}P \text { value for trend } \\
\text { (ANOVA) }\end{array}$} \\
\hline & & $\begin{array}{l}\text { Mean (SD) } \\
\text { IogMAR }\end{array}$ & $\begin{array}{l}\text { Mean difference }(95 \% \mathrm{Cl}) \\
\text { from no treatment }\end{array}$ & $\begin{array}{l}\text { Mean (SD) } \\
\text { IogMAR }\end{array}$ & $\begin{array}{l}\text { Mean difference }(95 \% \mathrm{CI}) \\
\text { from no treatment }\end{array}$ & \\
\hline \multicolumn{7}{|l|}{ At trial end point } \\
\hline Uncorrected acuity ( $\mathrm{n}=168)$ & $0.424(0.24)(n=56)$ & $0.381(0.23)(\mathrm{n}=56)$ & $0.043(-0.05$ to 0.13$)$ & $0.336(0.20)(n=56)$ & 0.088 (0.01 to 0.17$)$ & 0.041 \\
\hline Best corrected acuity ( $n=164)$ & $0.301(0.20)(n=55)$ & $0.216(0.17)(\mathrm{n}=55)$ & 0.085 (0.02 to 0.15$)$ & $0.193(0.12)(n=54)$ & 0.109 (0.05 to 0.17$)$ & 0.001 \\
\hline \multicolumn{7}{|l|}{ Six months after trial end } \\
\hline Best corrected acuity $(n=154)$ & $0.170(0.15)(n=50)$ & $0.197(0.16)(\mathrm{n}=51)$ & $0.03(-0.09$ to 0.03$)$ & $0.170(0.13)(n=53)$ & $0.0004(-0.06$ to 0.05$)$ & 0.996 \\
\hline
\end{tabular}

ANOVA=analysis of variance

Table 4 Visual acuity after follow up to trial end point and six months after trial, by treatment group and initial acuity

\begin{tabular}{|c|c|c|c|c|c|c|}
\hline & \multirow{2}{*}{$\begin{array}{c}\text { No treatment } \\
\text { Mean (SD) } \\
\text { logMAR }\end{array}$} & \multicolumn{2}{|c|}{ Glasses } & \multicolumn{2}{|c|}{ Full } & \multirow[b]{2}{*}{$\begin{array}{l}P \text { value for tren } \\
\text { (ANOVA) }\end{array}$} \\
\hline & & $\begin{array}{l}\text { Mean (SD) } \\
\text { IogMAR }\end{array}$ & $\begin{array}{l}\text { Mean difference }(95 \% \mathrm{Cl}) \\
\text { from no treatment }\end{array}$ & $\begin{array}{c}\text { Mean (SD) } \\
\text { IogMAR }\end{array}$ & $\begin{array}{l}\text { Mean difference }(95 \% \mathrm{Cl}) \\
\text { from no treatment }\end{array}$ & \\
\hline \multicolumn{7}{|l|}{ At end of trial } \\
\hline Mild $^{*}$ acuity loss ( $\left.n=101\right)$ & $0.22(0.17)(n=33)$ & $0.16(0.14)(n=35)$ & $0.058(-0.02$ to 0.13$)$ & $0.18(0.11)(\mathrm{n}=33)$ & $0.045(-0.02$ to 0.11$)$ & 0.11 \\
\hline Moderate† acuity loss ( $n=63$ ) & $0.42(0.19)(\mathrm{n}=22)$ & $0.31(0.17)(\mathrm{n}=20)$ & $0.112(-0.002$ to 0.23$)$ & $0.22(0.13)(n=21)$ & $0.203(0.10$ to 0.30$)$ & 0.0002 \\
\hline \multicolumn{7}{|l|}{ Six months after end of trial } \\
\hline Mild $^{*}$ acuity loss $(n=91)$ & $0.13(0.08)(n=28)$ & $0.13(0.12)(n=31)$ & $0.00(-0.06$ to 0.05$)$ & $0.16(0.12)(\mathrm{n}=32)$ & $-0.03(-0.08$ to 0.03$)$ & 0.327 \\
\hline Moderate† acuity loss ( $n=63)$ & $0.22(0.20)(n=22)$ & $0.30(0.18)(\mathrm{n}=20)$ & $-0.08(-0.19$ to 0.04$)$ & $0.19(0.14)(n=21)$ & $0.03(-0.07$ to 0.14$)$ & 0.575 \\
\hline
\end{tabular}

${ }^{*} 6 / 9$ or $6 / 12$ at presentation. $+6 / 18$ to $6 / 36$ at presentation.

powered to detect a clinically useful treatment effect, so it is unlikely that important treatment effects have been missed. It might be argued that the patching treatment regimens used were insufficiently aggressive, but these are the regimens in routine clinical use. ${ }^{27}$ The results of the trial should be generalisable to any centre treating children with unilateral visual impairment as we used standard screening criteria, recruited over two thirds of eligible children, and included a wide range of initial acuity levels. Although the age range in the trial was narrow, the effect of deferred treatment in the no treatment group indicates that these findings are also applicable to children identified at school entry. Their relevance to amblyopia secondary to squint is less clear.

\section{Responses to treatment}

The overall response to treatment was disappointing: an increase equivalent to one line on a Snellen chart. However, children with moderate initial acuity improved with full treatment from $6 / 18$ or worse to a mean acuity close to $6 / 9$, while those in the mild group were essentially unchanged. The benefit to acuity from 12 months of wearing glasses compared with no treatment suggests that the treatment effect from glasses alone is limited. ${ }^{19}$ The most striking effect was the additive effect of patching in the moderate acuity group.

As in all trials, there is the possibility that those left untreated may suffer, but the no treatment group in fact showed a tendency to spontaneous improvement. This is consistent with results from another study of amblyopia ${ }^{28}$ but contrasts with the results of an uncontrolled study, in which patients who did not comply with treatment deteriorated over time. ${ }^{29}$ However, it is well known that non-compliant people are not representative of patients in general and they tend to have a worse prognosis. Currently, continued wearing of glasses is recommended until the age of 7 , even if acuity improves to normal, to prevent the development of refractive amblyopia. ${ }^{30}$ Results in the untreated group, however, suggest that the risk of subsequently developing amblyopia is slight.

In many districts without preschool screening children are not detected or treated until school entry, the age at which our untreated group completed the trial and were offered treatment. The post-trial follow up shows that deferring their treatment did not limit their potential for improvement and nearly halved the proportion of children needing patching at all. This is consistent with another study that showed that presenting acuity, rather than age, is the most important determinant of outcome. ${ }^{6}$ The late results in the full group also suggest that the treatment effects persist well after most patching treatment has ceased. The glasses group with moderate initial acuity, in whom patching treatment was deferred, showed no overall gain in acuity at posttrial follow up. While this is probably a random effect, it raises the question whether prior refractive correction might in some way limit the effectiveness of subsequent patching.

\section{Conclusions}

Amblyopia and refractive error are common worldwide, and many countries have screening programmes to detect asymptomatic visual defects in children. Thus the implications of our findings are considerable and should provide helpful evidence for future service planning. Children with a moderate acuity loss of $6 / 18$ or worse showed a clear cut response to treatment, which

Table 5 Change in acuity after one year, by treatment group and initial acuity

\begin{tabular}{|c|c|c|c|c|c|c|}
\hline \multirow[b]{2}{*}{ Initial acuity category } & \multirow{2}{*}{$\begin{array}{c}\text { No treatment } \\
\text { Mean (SD) } \\
\text { logMAR }\end{array}$} & \multicolumn{2}{|c|}{ Glasses only } & \multicolumn{2}{|c|}{ Full } & \multirow[b]{2}{*}{$P$ value for trend } \\
\hline & & $\begin{array}{c}\text { Mean (SD) } \\
\text { logMAR }\end{array}$ & $\begin{array}{l}\text { Mean difference }(95 \% \mathrm{CI}) \\
\text { from no treatment }\end{array}$ & $\begin{array}{c}\text { Mean (SD) } \\
\text { logMAR }\end{array}$ & $\begin{array}{l}\text { Mean difference }(95 \% \mathrm{Cl}) \\
\text { from no treatment }\end{array}$ & \\
\hline \multicolumn{7}{|c|}{ Change in corrected acuity at 54 week follow up } \\
\hline Mild & $0.19(0.17)(n=30)$ & $0.24(0.14)(n=31)$ & $0.05(-0.03$ to 0.13$)$ & $0.23(0.17)(n=31)$ & $0.04(-0.06$ to 0.13$)$ & $0.38^{*}$ \\
\hline Moderate & $0.25(0.21)(\mathrm{n}=21)$ & $0.35(0.20)(\mathrm{n}=18)$ & $0.11(-0.03$ to 0.24$)$ & $0.52(0.19)(n=20)$ & $0.27(0.14$ to 0.39$)$ & $<0.001^{*}$ \\
\hline \multicolumn{7}{|c|}{ Worse (change $>0.1$ logMAR) uncorrected acuity at 52 weeks } \\
\hline Mild & $13 \%(4)$ & $6 \%(2)$ & - & $10 \%(3)$ & - & $0.28 \dagger$ \\
\hline Moderate & $24 \%(5)$ & $11 \%(2)$ & - & $15 \%(3)$ & - & $0.13 \dagger$ \\
\hline
\end{tabular}

${ }^{*}$ ANOVA. $\dagger \chi^{2}$. 


\section{What is already known on this topic}

Preschool vision screening aims to detect amblyopia at a stage when treatment is effective

Amblyopia has conventionally been treated with glasses, supplemented by patching of the better eye if necessary

Treated children tend to improve over time, but no study has included an untreated control group or compared outcomes for different levels of acuity at presentation

\section{What this study adds}

Treatment of children with considerably reduced acuity (6/18 and worse) can result in a mean acuity equivalent to $6 / 9$ on the Snellen chart

Children with $6 / 9$ or $6 / 12$ initial acuity show little benefit from treatment

Children whose treatment is deferred from age 4 until age 5 have the same acuity after treatment, but fewer need patching treatment at all

Over a third of children thought to require treatment after repeat screening do not have acuity loss

itself arguably justifies screening to identify and treat these children. In contrast, children with mild acuity loss, who represent over half those identified with unilateral acuity impairment at screening in this and other studies, ${ }^{31}$ received little benefit from either treatment. This level of impairment, though often excluded from studies, ${ }^{20}{ }^{25}$ is still commonly treated in routine clinical practice. We argue that children with $6 / 9$ in only one eye should no longer constitute screen failures and do not justify treatment, even with glasses.

Nearly $40 \%$ of the children referred for treatment did not in fact have the target condition. This was despite two tests in the community and presumably reflects difficulties in testing preschool children. This, together with the good response seen in those whose treatment was deferred, supports the use of relatively later screening, as recently suggested. ${ }^{32}$

We are grateful to the members of the data monitoring committee: S Stewart-Brown, A Fielder, and A MacIntyre; to all the staff from the trial centres: Helen Haggerty of Roval Victoria Infirmary, Newcastle upon Tyne; R W Allchin, Glenys Smithson, Tricia Allan, Jane Bogie, and Donna Longville of Sunderland Eye Infirmary; J Innes, S Rowe, S Medforth, J Eeles, L Thomas, and A Griffith of Hull Roval Infirmary; I Akroyd, D Heath, L James, C Doherty, D Dunham, and F Jackson of Liverpool Road Health Centre, Luton; N Astbury, T Salisbury, C Philpot, K Boling, and I Jandhu of Norfolk and Norwich University Hospital; G Bedford, H Cameron, S Armstrong, P Johnson, and A McLear of Dumfries and Galloway Roval Infirmary; P North, S Taylor, H Bowker, and N Thompson of Peel Health Centre, Bury; J Elston, H Wicks, J Ware, V Cardy, V Mehta, and C Jackson of Radcliffe Infirmary, Oxford; to all the children and parents who took part in the study; to N P Strong for facilitating and supporting this study; to P Griffiths for advice on the manuscript; and to J N S Matthews for statistical advice and for the randomisation software.

Contributors: MPC initiated, planned, and supervised the study and helped to draft the paper. CMW helped to plan the study, monitored adherence to the protocol, supervised the analysis, and helped to draft the paper. SH set up the study, trained trial participants, was responsible for day to day organisation, data entry, and analysis, and helped to draft the paper.JDA, JH, and SRR helped to plan and pilot the study, train the trial centres, and advised on trial organisation throughout. All authors have seen and commented on the final draft. MPC is guarantor for the study.

Funding: NHS research and development, Northern and Yorkshire: minimal role in study organisation apart from advising on size and length of trial.
Competing interests: None declared.

Ethical approval: The study was approved by the North West Multicentre Regional Ethical Committee and monitored by a data monitoring committee.

1 Hubel DH, Wiesel TN. The period of susceptibility to the physiological effects of unilateral eye closure in kittens. J Physiol 1970;206:419-36.

2 Barnes G, Hess R, Dumoulin S, Achtman R, Pike G. The cortical deficit in humans with strabismic amblyopia. J Physiol 2001;533:281-97.

3 Von Noorden GK, Campos EC. Binocular vision and ocular motility. 6th ed. St Louis, MO: Mosby, 2001.

4 Reeves B. Taxonomy and epidemiology of amblyopia. In: Moseley M, Fielder A, eds Amblyopia: a multidisciplinary approach. Oxford: Butterworth Heinemann, 2002:68-80.

Moseley M. Amblyopia: treatment and evaluation. In: Moseley M, Fielder A, eds. Amblyopia: a multidisciplinary approach. Oxford: Butterworth Heinemann, 2002:81-104.

Hardman-Lea SJ, Loades J, Rubinstein MP. The sensitive period for anisometropic amblyopia. Eye 1989;3:783-90.

7 Birnbaum M, Koslowe K, Sanet R. Success in amblyopia therapy as a function of age: a literature survey. Am J Optom Physiol Optics 1977;54:269-75.

8 Attebo K, Mitchell P, Cumming R, Smith W, Jolly N, Sparkes R. Prevalence and causes Attebo K, Mitchell P, Cumming R, Smith W, Jolly N, Sparkes R. Preva
of amblyopia in an adult population. Ophthalmology 1998;105:154-9.

9 Jakobsson P, Kvarnstrom G, Abrahamsson M, Bjernbrink-Hornblad E, Sunnqvist B. The frequency of amblyopia among visually impaired persons. Acta Ophthalmol Scand 2002;80:44-6.

10 Rahi J, Logan S, Timms C, Eggitt IR, Taylor DSI. Risk, causes, and outcomes of visual impairment after loss of vision in the non-amblyopic eye: a population based study. Lancet 2000;360:597-602.

11 Hartmann EE, Dobson V, Hainline L, Marsh-Tootle W, Quinn G, Ruttum MS, et al. Preschool vision screening: summary of a task force report. Ophthalmology 2001:108:479-85.

12 Beardsell R. Orthoptic visual screening at 3 s years by Huntingdon Health Authority. $\mathrm{Br}$ Orthopt J 1989;46:7-13.

13 Ingram RM, Holland WW, Walker C, Wilson JM, Arnold PE, Dally S. Screening for visual defects in preschool children. BrJ Ophthalmol 1986;70:16-21.

14 Taylor D. Screening for squint and poor vision [editorial]. Arch Dis Child 1987;62:982-3.

15 Snowdon SK, Stewart-Brown SL. Preschool vision screening. Health Technol Assess 1997;1:1-83.

16 Kvarnstrom G, Jakobsson P, Lennerstrand G. Visual screening of Swedish children: an ophthalmological evaluation. Acta Ophthalmol Scand 2001;79:240-4.

17 Kemper A, Margolis P, Downs S, Bordley W. A systematic review of vision screening tests for the detection of amblyopia. Pediatrics 1999;104:1220-2.

18 Roval College of Ophthalmologists, College of Optometrists, British Orthoptic Society Guidelines for children's eye care. London: Royal College of Ophthalmologists, Royal College of Anaesthetists, 2002.

19 Moseley M, Fielder A, Irwin M, Jones H, Auld R. Effectiveness of occlusion therapy in ametropic amblyopia: a pilot study. Br J Ophthalmol 1997;81:956-61.

20 Pediatric Eye Disease Investigator Group. A randomized controlled trial of atropine vs patching for treatment of moderate amblyopia in children. Arch Ophthalmol 2002;120:268-78.

21 Garey L, Blakemore C, Durand FV. Visual deprivation in monkeys: its effects and its reversal. Prog Brain Res 1979;51:445-56.

22 Stewart-Brown SL, Haslum MN, Butler N. Educational attainment of 10 year old children with treated and untreated visual defects. Dev Med Child Neurol 1985:27:504-13.

23 Stewart-Brown SL Haslum MN, Howlett B. Preschool vision screening: a service in need of rationalisation. Arch Dis Child 1988;63.356-9.

24 McGraw PV, Winn B. Glasgow acuity cards: a new test for the measurement of letter acuity in children. Ophthal Physiol Opt 1993;13:400-4.

25 Williams C, Northstone K, Harrad R, Sparrow J, Harvey I. Amblyopia treatment outcomes after screening before or at age 3 years: follow up from randomised trial. BMJ 2002;324:1549-51

26 Campos EC. Amblyopia. Surv Ophthalmol 1995;40:23-39.

27 Pediatric Eye Disease Investigator Group. A randomized trial of patching regimens for treatment of moderate amblyopia in children. Arch Ophthalmol 2003;121:603-11.

28 Hard AL, Williams P, Sjostrand J. Do we have optimal screening limits in Sweden for vision testing at the age of 4 years? Acta Ophthalmol Scand 1995;73:483-5.

29 Simons K, Preslan M. Natural history of amblyopia untreated due to lack of compliance. BrJ Ophthalmol 1999;83:582-7.

30 Kutschke P. Scott W, Keech R. Anisometropic amblyopia. Ophthalmology 1991;98:258-63.

31 Bray LC, Clarke MP, Jarvis SN, Francis PM, Colver A. Preschool vision screening: a prospective comparative evaluation. Eye 1996;10:714-8.

32 Hall D, Elliman D. Health for all children. Oxford: Oxford University Press, 2002.

(Accepted 24 September 2003)

bmj.com 2003;327:1251

Department of Ophthalmology, School of Neurobiology, Neurology and Psychiatry, University of Newcastle upon Tyne, Newcastle NE2 4HH

M P Clarke reader in ophthalmology

$\mathrm{S}$ Hrisos research associate

PEACH Unit, Department of Child Health, University of Glasgow, Glasgow G3 8SJ C M Wright senior lecturer in community child health

Orthoptic Department, Department of Ophthalmology, Royal Victoria Infirmary, Newcastle NE1 4LP

$\mathrm{J}$ D Anderson senior orthoptist

J Henderson head orthoptist

S R Richardson deputy head orthoptist

Correspondence to: M P Clarke

M.P.Clarke@ncl.ac.uk 